Bundesgesundheitsbl 2017 ·60:350-352

DOI 10.1007/s00103-016-2508-3

Online publiziert: 2. Januar 2017

๑) Springer-Verlag Berlin Heidelberg 2017
Empfehlung des Umweltbundesamtes

\section{Fortschreibung der vorläufigen Bewertung von per- und polyfluorierten Chemikalien (PFC) im Trinkwasser}

\author{
Empfehlung des Umweltbundesamtes nach \\ Anhörung der Trinkwasserkommission
}

\section{Anlass für diese Empfehlung}

Die Trinkwasserkommission des Bundesministeriums für Gesundheit (BMG) beim Umweltbundesamt hat zur Problematik der PFC im Trinkwasser zuletzt am 13.7.2006 in Form einer „vorläufige Bewertung von Perfluorierten Tensiden (PFT) im Trinkwasser am Beispiel ihrer Leitsubstanzen Perfluoroctansäure (PFOA) und Perfluoroctansulfonsäure (PFOS)“ Stellung genommen.

Seither sind sowohl zu PFOA und PFOS wie auch zu anderen PFC weitere Daten erarbeitet und veröffentlicht worden, die Anlass für eine Fortschreibung der damaligen vorläufigen Bewertung geben.

In Deutschland haben aktuell zu PFOA und PFOS die Kommission Human-Biomonitoring [1] des Umweltbundesamtes und, auch zu weiteren PFC, die LAWALABO-Kleingruppe „Ableitung von Geringfügigkeitsschwellenwerten für PFC“ des Ständigen Ausschusses „Grundwasser und Wasserversorgung" der LAWA (LAWA-LABO-Kleingruppe PFC) humantoxikologische Bewertungen erarbeitet. Auf diese Bewertungen gründen die vorliegenden Empfehlungen.

\section{Empfehlungen}

Die LAWA-LABO-Kleingruppe PFC hat aus Informationen zu Vorkommen und Verbreitung sowie aus Einzelfallberichten 13 PFC als für das Grundwasser prioritär benannt. Für sieben dieser 13 PFC erster
Priorität war die Datenlage ausreichend, um einen Leitwert nach den Kriterien der Trinkwasserverordnung abzuleiten. Die Bewertung von Perfluorhexansulfonsäure (PFHxS) wurde aber als grenzwertig angesehen. Ihr liegt nicht eine 90-Tages-Studie als übliches Mindestkriterium zugrunde, sondern eine Studie mit nur 42 Tagen Expositionszeit. Besonders vor dem Hintergrund des Bedarfs für Leitwerte wurde dieses Bewertungsergebnis noch akzeptiert.

Die Ergebnisse einer umfassenden Neubewertung im Sinne von Trinkwasser-Leitwerten $\left(\mathrm{TW}_{\mathrm{LW}}\right)$ oder Gesundheitlichen Orientierungswerten (GOW) zeigt nachstehende Tabelle.

Der deutlich niedrigste Wert ergibt sich für Perfluornonansäure (PFNA) mit $60 \mathrm{ng} / \mathrm{l}$. In ihm ist allerdings wegen des reproduktionstoxischen (Einstufung Repr. 1B) und vermutlich krebserzeugenden (Einstufung Carc. 2) Potentials ein besonderer Sicherheitsfaktor von zehn eingerechnet. Als bisher höchsten Wert ergibt sich $10 \mu \mathrm{g} / \mathrm{l}$ für Perfluorbutansäure (PFBA), der damit etwas höher liegt als der bisher vom UBA genannte Wert [2] $(7 \mu \mathrm{g} / \mathrm{l})$. Die verschiedenen Werte zu den einzelnen Verbindungen scheinen darüber hinaus in einem vernünftigen Verhältnis zueinander zu stehen, das ihre Struktur (Kettenlänge) widerspiegelt.

Bei Stoffen, für die keine ausreichenden Daten für eine humantoxikologische Bewertung für einen $\mathrm{TW}_{\mathrm{LW}}$ vorlagen, wurde hilfsweise das vom Umweltbun- desamt für die Bewertung von humantoxikologisch nur teil- oder nicht bewertbaren Stoffen entwickelte Konzept der Gesundheitlichen Orientierungswerte (GOW) angewendet. GOW werden über Evidenzkriterien (z. B. gentoxisch ja oder nein) und Erfahrungswissen für ihre Höhe begründet [3]. Sie stellen insoweit semiquantitative Bewertungsergebnisse dar, die zwar besondere Unsicherheiten enthalten, im Grundsatz aber als protektiv anzusehen sind. GOW werden grundsätzlich stoffspezifisch und fallunabhängig abgeleitet. Werden sie in Einzelfällen überschritten, sollte das Gesundheitsamt im Zusammenhang mit Überlegungen zu Minderungs- oder Abhilfemaßnahmen neben ihrer fachlichen Grundlage auch stoffspezifische Besonderheiten (im Falle der PFC z. B. die biologischen Halbwertszeiten) und möglicherweise weitere Beurteilungskriterien bei der Einzelfallbetrachtung berücksichtigen.

PFC werden weder zur Gewinnung und Verteilung von Trinkwasser benötigt noch gehören sie zu seinen natürlichen Bestandteilen. Es sind Verunreinigungen, die die Beschaffenheit des Trinkwassers nachteilig beeinflussen und sie sind in ihrer Konzentration nach dem Minimierungsgebot gemäß $\$ 6$ Absatz 3 TrinkwV 2001 so niedrig zu halten, wie dies nach den Umständen des Einzelfalles auf Grundlage der allgemein anerkannten Regeln der Technik (aaRdT) möglich erscheint. Das Trinkwasser soll i.S. von $\$ 1$ TrinkwV 2001 die Verbraucher uneinge- 


\begin{tabular}{|c|c|c|c|}
\hline $\begin{array}{l}\text { Lfd. } \\
\text { Nr. }\end{array}$ & Name, Abkürzung (CAS Nr.) & $\begin{array}{l}\mathrm{TW}_{\mathrm{LW}} \\
{[\mu \mathrm{g} / \mathrm{l}]}\end{array}$ & $\begin{array}{l}\text { GOW } \\
{[\mu \mathrm{g} / \mathrm{l}]}\end{array}$ \\
\hline 1 & Perfluorbutansäure, PFBA (375-22-4) & 10 & - \\
\hline 2 & Perfluorpentansäure, PFPeA (2706-90-3) & - & 3,0 \\
\hline 3 & Perfluorhexansäure, PFHxA (307-24-4) & 6 & - \\
\hline 4 & Perfluorheptansäure, PFHpA (375-85-9) & - & 0,3 \\
\hline 5 & Perfluoroktansäure, PFOA (335-67-1) & 0,1 & - \\
\hline 6 & Perfluornonansäure, PFNA (375-95-1) & 0,06 & - \\
\hline 7 & Perfluordekansäure, PFDA (335-76-2) & - & 0,1 \\
\hline 8 & Perfluorbutansulfonsäure, PFBS (375-73-5) & 6 & - \\
\hline 9 & Perfluorhexansulfonsäure, PFHxS (355-46-4) & 0,1 & - \\
\hline 10 & Perfluorheptansulfonsäure, PFHpS (375-92-8) & - & 0,3 \\
\hline 11 & Perfluoroktansulfonat, PFOS (1763-23-1) & 0,1 & - \\
\hline 12 & H4-Polyfluoroktansulfonsäure, H4PFOS (27619-97-2) & - & 0,1 \\
\hline 13 & Perfluoroktansulfonamid, PFOSA (754-91-6) & - & 0,1 \\
\hline
\end{tabular}

schränkt genusstauglich und so rein wie möglich erreichen.

\section{Unsicherheitsanalyse}

Die LAWA-LABO-Kleingruppe PFC führt in ihren Vorbemerkungen aus, dass die humantoxikologischen Bewertungen hauptsächlich auf vorliegenden Bewertungen anderer Institutionen basieren. Wenn solche Bewertungen nicht vorlagen, wurden die jeweiligen PFC anhand einzelner Studien bewertet. Dabei lag der Schwerpunkt auf der für eine Wertbegründung relevanten Ableitung; es war hier nicht vorgesehen, das gesamte Wissen zu den PFC-Wirkungen synoptisch darzustellen.

Von den sieben PFC, für die Werte analog der Trinkwasserverordnung abgeleitet werden konnten, lag nur für eine Substanz für die Schlüsselstudie der Mindestdatensatz einer (subchronischen) 90-Tages-Studie vor. Ansonsten waren die Schlüsselstudien mit mindestens doppelt so langer Expositionszeit, zweimal über zwei Jahre, durchgeführt worden und zweimal lag eine 2-Generationen-Studie vor. Insoweit können diese Ableitungen als ausreichend sicher angesehen werden. Als kritisch muss die Datenbasis zu PFHxS angesehen werden, da hier die Expositionszeit mit 42 Tagen unter dem geforderten subchronischen Test (90 Tage) lag. Da die GOW-Betrachtung das Bewertungsergebnis stützt, erscheint hier eine Risikoun- terschätzung aber wenig wahrscheinlich, so dass der resultierende Wert als $\mathrm{TW}_{\mathrm{LW}}$ akzeptiert werden kann.

Verschiedene Bewertungen andere Institutionen sehen besondere Sicherheitsfaktoren wegen fehlender z.B. entwicklungstoxikologischer und/oder immuntoxikologischer Daten vor. Dies ist bei einer Bewertung analog der Trinkwasserverordnung nicht vorgesehen, wodurch die Möglichkeit einer Risikounterschätzung gegeben sein kann. Im Zusammenhang mit den PFC reagiert der Mensch andererseits weniger empfindlich auf die PPARa-agonistische Wirkung als Ratte und Maus; Affen, die ebenfalls weniger empfindlich auf PPARa-Agonisten reagieren, wären ein passenderes Modell für den Menschen [4]. Die Ableitung humantoxikologisch begründeter Werte analog Trinkwasserverordnung von Nager-Daten könnte daher zu vorsichtigeren Werten führen.

GOW werden mangels sonstiger geeigneter Daten über Evidenzkriterien (z. B. gentoxisch ja oder nein) und Erfahrungswissen für ihre Höhe begründet [5]. Sie stellen insoweit semiquantitative Bewertungsergebnisse dar, die zwar besondere Unsicherheiten enthalten, im Grundsatz aber als protektiv anzusehen sind. GOW werden daher aber grundsätzlich nicht für möglicherweise maßnahmenbewehrte allgemein gültige Situationen oder Szenarien abgeleitet, sondern für Einzelfälle, in denen ihr Charakter, die besonderen Rahmenbedingungen des Falles und mögli- cherweise weitere Beurteilungskriterien berücksichtigt werden müssen. Aus gleichen Gründen wird auch empfohlen für die gelegentlich geforderte Bewertung des Vorkommens mehrere PFC nach der Additionsregel die GOW nicht mit einzubeziehen. Da verschiedenen PFC bedingt durch lange Halbwertszeiten im menschlichen Körper kumulieren können, besteht hinsichtlich der hier genannten GOW, die dieses Kriterium üblicherweise nicht einrechnen, eine besondere Unsicherheit. Dies kann zu Risikounterschätzungen führen und ist daher im Einzelfall ihrer Verwendung besonders zu beachten.

\section{Begründungen}

Für die vorgeschlagenen Werte werden die Begründungen der LAWA-LABOKleingruppe PFC übernommen. Sie verweist hinsichtlich einer ausführlichen und aktuellen allgemeinen Charakterisierung der PFC und ihrer Toxizität auf die U.S.Agency for Toxic Substances and Disease Registry [4] (hauptsächlich gestützt auf Daten zu PFOA und PFOS) und fasst folgendermaßen zusammen:

Epidemiologische Studien zeigen statistisch signifikante Zusammenhänge der Konzentrationen von PFC im Serum (besonders für PFOA und PFOS) und einer Vielzahl von auch konzentrationsabhängigen Gesundheitseffekten, wenn diese auch nicht immer über die verschiedenen Studien konsistent auftraten. Konsistent waren Assoziationen des Serumspiegels für PFOA und PFOS mit erhöhter Lipidkonzentration im Serum, erhöhter Harnsäure, reduziertem Geburtsgewicht, und Modifizierungen von Biomarkern für Leberschäden. Es gibt auch den Verdacht (equivocal evidence) auf Kanzerogenität.

Hinsichtlich der Biomarker von möglichen Leber-Effekten zeigte sich in Arbeitsplatzstudien keine konsistente Assoziation zwischen Serum-Leber-Enzymen (in erster Linie Alanin-Aminotransferase, ALT, Aspartat-Aminotransferase, AST, und $\gamma$-Glutamyltransferase, GGT) und der PFOA- oder PFOS-Konzentration im Serum. Eine Studie mit hoch PFOA-exponierten Probanden der Allgemeinbevölkerung fand signifikante Zusammenhänge zwischen PFOA- und PFOS-Serumkonzentrationen und ALT- 
sowie Bilirubin-Konzentrationen, wobei das Ausmaß als wahrscheinlich nicht biologisch relevant bewertet wurde. Studien mit Ratten, Mäusen und Affen haben die Leber als eines der toxikologisch empfindlichsten Zielorgane identifiziert. Die Daten zum Menschen sind dagegen nicht so aussagekräftig, wobei die PFOA- und PFOS-Serumkonzentrationen hier deutlich niedriger lagen als die, die in den Versuchstieren zu den Effekten führten.

Viele der in Versuchstieren beobachteten adversen Gesundheitseffekte werden der Fähigkeit der PFC zugeschrieben, den Peroxisomen-Proliferator-aktivierten Rezeptor- $\alpha$ (PPAR $\alpha$ ) zu aktivieren. PPAR $\alpha$ reguliert einige physiologische Prozesse, unter anderem auch die Fettsäure-Oxidation in der Leber; ihm wird auch Einfluss auf Reproduktion und Entwicklung zugeschrieben [6] und er wird mit der TumorInduktion in der Leber von Nagetieren durch nicht-gentoxische Karzinogene in Verbindung gebracht [7]. Es wurden Speziesunterschiede in der Reaktion auf PPARaAgonisten gefunden: Ratten und Mäuse sind die empfindlichsten Arten, Versuchskaninchen, nicht menschliche Primate und der Mensch sind weniger empfindlich. Erklärungen könnten z.B. Unterschiede in der Induzierbarkeit des PPARa durch die Exposition gegen einen Peroxisomenproliferator oder Unterschiede in einer gewebespezifischen Expression des PPARa sein. Nach der Aktivierung des Rezeptors in Nagetieren beginnt, hauptsächlich aber nicht nur in der Leber, eine charakteristische Folge von morphologischen und biochemischen Ereignissen. Dies schließt eine hepatozelluläre Hypertrophie durch die Zunahme von Zahl und Größe der Peroxisomen, einer starken Zunahme der peroxisomalen Fettsäure- $\beta$-Oxydation, eine erhöhte CYP450-vermittelte $\omega$-Hydroxylierung von Laurinsäure sowie Modifizierungen im Lipid-Metabolismus ein. Studien mit PPARa-Null-Mäusen zeigten allerdings auch von PPARa unabhängige Mechanismen der PFOA- und PFOS-Toxizität, einschließlich ihrer Lebertoxizität (Hepatomegalie in Mäusen des Wildtyps und in PPARa-Null-Mäusen im gleichen Ausmaß, wobei eine Aktivitätserhöhung der Acyl-Coenzym-A-Oxidase in den PPARa-Null-Mäusen fehlte). In einer Erhöhung des absoluten Lebergewichts re- sultierte auch eine PFOA-Exposition von Affen, zum Teil assoziiert mit einer signifikanten mitochondrialen, nicht aber mit einer peroxisomalen Proliferation.

Eine Erhöhung des Lebergewichts (und der Parameter der Fettsäure- $\beta$ Oxydation) zeigte sich allgemein verstärkt bis zu einer PFC-Kettenlänge von ungefähr zehn Kohlenstoffatomen. Eine signifikante peroxisomale Aktivität scheint eine Kohlenstoffkettenlänge von größer sieben zu erfordern, wobei eine Erhöhung über das Kontrollniveau hinaus schon bei Kettenlänge mit vier Kohlenstoffatomen berichtet wurden. Es zeigte sich auch, dass die unterschiedliche Aktivität selbst nicht direkt mit der Kohlenstoffkettenlänge verbunden ist, wohl aber mit einer unterschiedlichen Akkumulation in der Leber.

Wie zu PFOA und PFOS wurden auch zu PFDA in Mäusen Effekte hinsichtlich einer Zunahme der fötalen Mortalität beobachtet. Im Gegensatz dazu zeigte die orale Exposition von PFBA oder PFHxS keinen Effekt auf das Überleben oder das Körpergewicht von Nachkommen. Eine reduzierte spontane Aktivität von Mäusen, gefolgt von einer erhöhten Aktivität, wurde wiederum beobachtet, wenn sie bis zum 10 postnatalen Tag gegen $\mathrm{PFHxS}$ exponiert waren; keine derartigen Effekte zeigten gegen PFDA vergleichbar exponierte Mäuse.

Studien zur Immuntoxizität von PFC in Ratten und Mäusen weisen auf eine deutlich höhere Empfindlichkeit der Mäuse gegenüber den Ratten. Die durch PFOA und PFOS in adulten Mäusen induzierten immunologischen Veränderungen waren durch Thymus- und Milz-Atrophie, phänotypische Veränderungen der Thymusund Milzzellen und Beeinträchtigung der Reaktion auf sogenannte T-abhängige Antigene charakterisiert.

PFOA induziert wie viele andere PPARa-Agonisten in Ratten Hepato- und Leydig-Zelladenome sowie Adenome der Azinuszellen der Bauchspeicheldrüse. Nach einer umfassende Würdigung der Literatur wurde geschlussfolgert, dass der Mensch, obwohl er PPARa in genügender Menge für eine hypolipidische Reaktion auf therapeutische Arzneimittel besitzt, sich qualitativ und quantitative ausreichend hinsichtlich der Reaktion seiner Leber gegenüber PPARa-Agonisten im
Vergleich zu der von Ratten unterscheidet (wegen Unterschieden bei Genpromotoren, Rezeptoraktivität und -dichte), so dass der Wirkmechanismus für Lebertumoren in Tieren im Menschen so wahrscheinlich nicht funktioniert. Für die U.S.-ATSDR bleiben wegen ungenügender Daten jedoch Unsicherheiten, ob der Wirkmechanismus der PPARa-Agonisten für eine Risikobewertung auf den Menschen nicht übertragbar ist.

Die Begründungen der vorgeschlagenen Werte im Einzelnen sind der Netzseite des Umweltbundesamtes zu entnehmen (https://www.umweltbundesamt.de/ themen/wasser/trinkwasser/rechtlichegrundlagen-empfehlungen-regelwerk/ empfehlungen-stellungnahmen-zu-trinkwasser).

\section{Literatur}

1. Kommission Human-Biomonitoring. http://www. umweltbundesamt.de/themen/gesundheit/ kommissionen-arbeitsgruppen/kommissionhuman-biomonitoring. Zugegriffen: 23. Dez. 2016

2. UBA, Umweltbundesamt (2011) Grenzwerte, Leitwerte, Orientierungswerte, Maßnahmenwerte - Aktuelle Definitionen und Höchstwerte. http:// www.umweltbundesamt.de/sites/default/files/ medien/377/dokumente/grenzwerte_leitwerte. pdf. Zugegriffen: 23. Dez. 2016

3. Grummt T, Kuckelkorn J, Bahlmann A et al (2013) Tox-Box: Securing drops of life - an enhanced health-related approach for risk assessment of drinking water in Germany Tox-Box: Die Tropfen des Lebens bewahren - Gesundheitsbasierte Risikobewertung für Trinkwasser in Deutschland. Environ Sci Eur 25:27-34

4. Agency for Toxic Substances and Disease Registry (2015) Toxicological Profile for Perfluoroalkyls, Draft for Public Com-ment. http://www.atsdr.cdc. gov/ToxProfiles/tp200.pdf. Zugegriffen: 23. Dez. 2016

5. Grummt T, Kuckelkorn J, Bahlmann A et al (2013) Tox-Box: Securing drops of life - an enhanced health-related approach for risk assessment of drinking water in Germany Tox-Box: Die Tropfen des Lebens bewahren - Gesundheitsbasierte Risikobewertung für Trinkwasser in Deutschland. Environ Sci Eur 25:27-34

6. Abbott BD (2009) Review of the expression of peroxisome proliferator-activated receptors alpha (PPAR alpha), beta (PPAR beta), and gamma (PPAR gamma) in rodent and human development. Reprod Toxicol 27:246-257

7. Klaunig JE, Babich MA, Baetcke KP, Cook JC, Corton JC, David RM, DeLuca JG, Lai DY, McKee RH, Peters JM, Roberts RA, Fenner-Crisp PA (2003) PPARalpha agonist-induced rodent tumors: Modes of action and human relevance. Crit Rev Toxicol 33:655-780 